\title{
A MODIFIED BROADCAST STRATEGY FOR DISTRIBUTED SIGNAL ESTIMATION IN A WIRELESS SENSOR NETWORK WITH A TREE TOPOLOGY
}

\author{
Joseph Szurley ${ }^{\S}$, Alexander Bertrand ${ }^{\S}$, Marc Moonen ${ }^{\S}$ \\ $\S K U$ Leuven-Dept. Electrical Engineering ESAT \\ STADIUS Center for Dynamical Systems, \\ Signal Processing and Data Analytics \\ Kasteelpark Arenberg 10, 3001 Leuven, Belgium \\ E-mail: joseph.szurley, alexander.bertrand, \\ marc.moonen@esat.kuleuven.be
}

\begin{abstract}
We envisage a wireless sensor network (WSN) where each node is tasked with estimating a set of node-specific desired signals that has been corrupted by additive noise. The nodes accomplish this estimation by means of the distributed adaptive node-specific estimation (DANSE) algorithm in a tree topology (T-DANSE). In this paper, we consider a network where there is at least one node with a large (virtually infinite) energy budget, which we select as the root node. We propose a modification to the signal flow of the T-DANSE algorithm where instead of each node having two-way signal communication, there is a single signal flow toward the root node of the tree topology which then broadcasts a single signal to all other nodes. We demonstrate that the modified algorithm is equivalent to the original T-DANSE algorithm in terms of the signal estimation performance, shifts a large part of the communication burden toward the highpower root node to reduce the energy consumption in the low-power nodes and reduces the input-output delay.
\end{abstract}

Index Terms- Wireless sensor networks, distributed estimation, tree topology

\section{INTRODUCTION}

A wireless sensor network (WSN) consists of a set of nodes that contain sensors and are distributed over an environment in order to estimate a desired signal or parameter. There has been a rapid increase in their use in many applications $[1,2]$ which is due, in part, to the relative ease of deployment and reduction in cost on a per node level, while still maintaining the same performance of larger, more sophisticated wired sensing networks [3]. The nodes that form a WSN typically accomplish a network-wide task by cooperative communi-

This research work was carried out at the ESAT Laboratory of KU Leuven, in the frame of KU Leuven Research Council CoE EF/05/006 'Optimization in Engineering' (OPTEC) and PFV/10/002 (OPTEC), Concerted Research Action GOA-MaNet, the Belgian Programme on Interuniversity Attraction Poles initiated by the Belgian Federal Science Policy Office: IUAP P7/23 'Belgian network on stochastic modeling analysis design and optimization of communication systems' (BESTCOM) 2012-2017, Research Project iMinds, Research Project FWO nr. G.0763.12 'Wireless Acoustic Sensor Networks for Extended Auditory Communication' and project HANDiCAMS. The project HANDiCAMS acknowledges the financial support of the Future and Emerging Technologies (FET) programme within the Seventh Framework Programme for Research of the European Commission, under FET-Open grant number: 323944. Alexander Bertrand is supported by a Postdoctoral Fellowship of the Research Foundation Flanders (FWO). The scientific responsibility is assumed by its authors.

\author{
Ingrid Moerman ${ }^{\ddagger}$
}

\author{
$\ddagger$ Ghent University \\ Department of Information Technology (INTEC), \\ iMinds Future Health Department \\ Gaston Crommenlaan 8, Bus 201, \\ 9050 Ghent, Belgium \\ E-mail: ingrid.moerman@intec.ugent.be
}

cation where each node collects a local set of measurements that are aggregated with information from neighboring nodes.

In the envisaged WSN each node is tasked with estimating a set of node-specific desired signals that have been corrupted by additive noise. We assume that the nodes are equipped with local processing capabilities and have a finite energy budget, except for a designated root node which will be defined in the sequel. They perform an estimation of their desired signals by finding the linear minimum mean squared error (LMMSE) between their desired signals and a linear filtered version of their sensor signals. In order to improve the LMMSE estimates, nodes also collect sensor signals that are transmitted by other nodes in the WSN.

However, to find the network-wide optimal estimates each node would need to receive all the sensor signals of all other nodes in the WSN which would require either a direct connection between nodes or the use of relays $[4,5]$ to ensure that the signals are diffused through the entire WSN. This may not only use a significant amount of bandwidth, but may also require a substantial amount of transmission energy if there is a large distance between nodes.

It is with this in mind that we employ the distributed adaptive node-specific signal estimation algorithm in a tree topology (TDANSE) [6],[7]. In the T-DANSE algorithm, each node uses its own sensor signals and fuses these together with the received signals from other nodes. This signal fusion reduces the amount of bandwidth required for the transmission of each node's signal. Furthermore, in using a tree topology, each node needs only to communicate with nearby nodes which can reduce the transmission energy [8].

The T-DANSE algorithm has two modes of signal flow : a fusion flow where signals travel from the outer most nodes or leaf nodes which have a single neighbor, toward the root node; and a diffusion flow, where the signals travel away from the root node. However, this entails that each non-root and non-leaf node transmits two signals, one for each signal flow, which uses a significant portion of the already limited energy resources.

In this paper, we look to modify the signal data flow between the nodes in the T-DANSE algorithm. The fusion flow is equivalent to the original T-DANSE algorithm, however, the diffusion flow is now replaced with a single broadcast signal from the root node. This is shown to shift the majority of energy usage for signal transmission to the root node, thereby reducing the energy usage of nonroot nodes, which lends itself to such applications as heterogeneous WSNs where it is assumed that the sink node typically has larger communication and energy capabilities $[9,10]$. In many applications it is assumed that the sink node already communicates directly to the other nodes $[9,11]$, therefore this type of broadcasting exploits this 
type of connectivity. The proposed data flow is also shown to reduce the input-output delay in the tree topology.

This paper is organized as follows: Section 2 introduces the network and data model for the WSN. Section 3 reviews the T-DANSE algorithm highlighting the fusion and diffusion signal flow of the WSN. Section 4 introduces the proposed modification to the signal flow of the T-DANSE algorithm and discusses the equivalence in terms of signal estimation performance to the original algorithm. Section 5 shows the convergence properties of the proposed algorithm and shift in energy to that of the root node from the proposed signal flow. Finally, conclusions are given in Section 6.

\section{DATA MODEL}

A WSN is envisaged in a tree topology consisting of $K$ nodes. We assume a perfect bi-directional wireless link between neighboring nodes. The set of neighbors of node $\mathrm{k}$, i.e., the nodes with which node $k$ can communicate, is denoted by $\mathcal{N}_{k}$.

Node $k$ collects an $M_{k}$-channel signal, $\mathbf{y}_{k}$, corresponding to sensors signals at node $k$, from which the $m^{t h}$ channel is given as

$$
y_{k, m}[t]=x_{k, m}[t]+n_{k, m}[t], m \in\left\{1, \ldots, M_{k}\right\}
$$

where $t$ is the discrete time index, $x_{k, m}[t]$ is the desired signal component and $n_{k, m}[t]$ is additive noise. We assume that the collected signals are complex allowing for a frequency domain description where, for the sake of brevity, we omit the discrete time index from the following analysis.

The desired signal components, $\mathbf{x}_{k}=\left[x_{k, 1}, \ldots, x_{k, M_{k}}\right]^{T}$, of the sensor signals of a node consist of a linear combination of $Q$ source signals, stacked in the $Q$-channel signal $\mathbf{s}$, and mixed by an $M_{k} \times Q$ steering matrix $\left(Q \leq M_{k}\right)$, $\mathbf{A}_{k}$, i.e.,

$$
\mathbf{x}_{k}=\mathbf{A}_{k} \mathbf{S}
$$

where it is assumed that $\mathbf{A}_{k}, \forall k \in K$.

The goal of each node is to estimate the local desired signal components $\mathbf{x}_{k}$ in the local sensors. Without loss of generality, we assume that each node $k$ estimates only the first $Q$ signals in $\mathbf{x}_{k}{ }^{1}$. We then define the $Q$-channel signal $\overline{\mathbf{x}}_{k}=\overline{\mathbf{A}}_{k}$ s, where $\overline{\mathbf{A}}_{k}$ is a $Q \times Q$ submatrix of $\mathbf{A}_{k}$ containing the first $Q$ rows of $\mathbf{A}_{k}$.

In a centralized scenario, where all nodes have access to all sensor signals, node $k$ estimates $\overline{\mathbf{x}}_{k}$ by applying a linear estimator to the received sensor signals $\mathbf{W}_{k}^{H} \mathbf{y}$, where ${ }^{H}$ is the conjugate transpose and $\mathbf{y}=\left[\mathbf{y}_{1}^{T} \ldots \mathbf{y}_{K}^{T}\right]$. Node $k$ finds $\mathbf{W}_{k}$ by performing a LMMSE in the form of

$$
\hat{\mathbf{W}}_{k}=\underset{\mathbf{W}_{k}}{\arg \min } E\left\{\left\|\overline{\mathbf{x}}_{k}-\mathbf{W}_{k}^{H} \mathbf{y}\right\|_{2}^{2}\right\}
$$

where $E\{$.$\} denotes the mathematical expectation. The linear esti-$ mator can be partitioned as $\mathbf{W}_{k}=\left[\mathbf{W}_{k 1}^{T} \ldots \mathbf{W}_{k K}^{T}\right]$ where $\mathbf{W}_{k q}$ is applied to the sensor signals of a node $\mathbf{y}_{q}$. The solution to (3) is the multi-channel Wiener filter (MWF)

$$
\hat{\mathbf{W}}_{k}=\mathbf{R}_{\mathbf{y} \mathbf{y}}^{-1} \mathbf{R}_{\mathbf{x} \mathbf{x}} \mathbf{E}_{K}
$$

where $\mathbf{R}_{\mathbf{y} \mathbf{y}}=E\left\{\mathbf{y} \mathbf{y}^{H}\right\}, \mathbf{R}_{\mathbf{x} \mathbf{x}}=E\left\{\mathbf{x} \mathbf{x}^{H}\right\}$ and $\mathbf{E}_{K}$ is an $M \times Q$ matrix where each column has a single entry equal to 1 that corresponds to a different desired sensor signal and 0 elsewhere. Using

\footnotetext{
${ }^{1}$ It can be shown that, due to the underlying $Q$-dimensional desired signal subspace (see (2)), the other signals in $\mathbf{x}_{k}$ can then also be optimally estimated from the signals obtained after convergence of the T-DANSE algorithm based on the estimation of the first $Q$ signals in each node [7].
}

(2) and the relationship between node-specific desired signals, the optimal filters at each node can be shown to be related by

$$
\forall k, q \in K, \hat{\mathbf{W}}_{k}=\hat{\mathbf{W}}_{q} \overline{\mathbf{A}}_{k q}
$$

where $\overline{\mathbf{A}}_{k q}=\overline{\mathbf{A}}_{q}^{-H} \overline{\mathbf{A}}_{k}^{H}$.

\section{THE T-DANSE ALGORITHM}

The nodes now look to estimate their node-specific desired signals by using the distributed adaptive node-specific estimation algorithm in a tree topology (T-DANSE) ${ }^{2}$ where each node uses its own sensor signals and fuses these together with the received signals from neighboring nodes.

Node $k$ collects its own sensor signals, $\mathbf{y}_{k}$, and receives fused $Q$-channel signals, $\mathbf{z}_{q k}, q \in \mathcal{N}_{k}$, from its neighboring nodes which have yet to be defined (see (9)-(11)), where the subscript $q k$ indicates the signal is from node $q$ and sent to node $k$. The received signals of the neighboring nodes, $\mathbf{z}_{q k}, \forall q \in \mathcal{N}_{k}$, are placed in a stacked vector $\mathbf{z}_{k_{-k}}$ where the subscript $-k$ indicates there is no $\mathbf{z}_{k k}$ signal, as the nodes themselves have access to their own sensor signals. Node $k$ places both its own sensor signals and the received signal from other nodes in a stacked vector given as $\tilde{\mathbf{y}}_{k}=\left[\mathbf{y}_{k}^{T} \mathbf{z}_{k_{-k}}^{T}\right]^{T}$. The desired signal components are also denoted in a similar fashion as $\tilde{\mathbf{x}}_{k}$.

We define the node-specific estimator that is applied in node $k$ as $\widetilde{\mathbf{W}}_{k}$, which is found in an iterative fashion by solving the local node-specific LMMSE problem,

$$
\widetilde{\mathbf{W}}_{k}^{i+1}=\underset{\widetilde{\mathbf{W}}_{k}}{\arg \min } E\left\{\left\|\overline{\mathbf{x}}_{k}-\widetilde{\mathbf{W}}_{k}^{H} \tilde{\mathbf{y}}_{k}^{i}\right\|_{2}^{2}\right\}
$$

where $i$ is the iteration index. We note that the $\tilde{\mathbf{y}}$ is updated at each iteration due to the fusion rules that generate the transmitted signals, which are defined in (9)-(11). The node-specific estimator consists of two components, one applied to the nodes sensor signals, $\mathbf{W}_{k k}$, and another that is applied to the received signals $\mathbf{z}_{k_{-k}}, \mathbf{G}_{k_{-k}}^{i+1}$.

The solution to (6) is again given as a MWF, which using the described partitioning, is

$$
\left[\begin{array}{l}
\mathbf{W}_{k k}^{i+1} \\
\mathbf{G}_{k_{-k}}^{i+1}
\end{array}\right]=\mathbf{R}_{\tilde{\mathbf{y}}_{k}^{i} \tilde{\mathbf{y}}_{k}^{i}}^{-1} \mathbf{R}_{\tilde{\mathbf{x}}_{k}^{i} \tilde{\mathbf{x}}_{k}^{i}} \mathbf{E}_{K}
$$

where $\mathbf{R}_{\tilde{\mathbf{y}}_{k}^{i} \tilde{\mathbf{y}}_{k}^{i}}=E\left\{\tilde{\mathbf{y}}_{k}^{i} \tilde{\mathbf{y}}_{k}^{i H}\right\}, \mathbf{R}_{\tilde{\mathbf{x}}_{k}^{i} \tilde{\mathbf{x}}_{k}^{i}}=E\left\{\tilde{\mathbf{x}}_{k}^{i} \tilde{\mathbf{x}}_{k}^{i H}\right\}$ and $\mathbf{E}_{K}$ is now an $\left|\tilde{\mathbf{x}}_{k}\right| \times Q$ matrix defined in Section 2 .

The estimated signal at node $k$ and at iteration $i$ is given as

$$
\overline{\mathbf{x}}_{k}^{i}=\left(\mathbf{W}_{k k}^{i+1}\right)^{H} \mathbf{y}_{k}^{i}+\left(\mathbf{G}_{k_{-k}}^{i+1}\right)^{H} \mathbf{z}_{k_{-k}}^{i} .
$$

In order for information to pass through the WSN, we define two types of data flow: a fusion flow which is initiated at the leaf nodes and travels inwards to a pre-defined root node, and a diffusion flow which is initiated at the root node once it receives all of it neighboring nodes signals and which travels outwards to the leaf nodes.

\subsection{Fusion flow}

The fusion flow is initiated when new sensor observations become available at the leaf nodes, which transmit a linear combination of their sensor signals to their single neighbor, i.e.,

$$
\mathbf{z}_{k q}^{i}=\mathbf{W}_{k k}^{i H} \mathbf{y}_{k}^{i}
$$

${ }^{2}$ It should be noted that the following description is only meant to be a succinct summary of the T-DANSE algorithm and the reader should refer to [7] for a more in depth discussion as well as convergence proofs. 
where $\mathbf{W}_{k k}^{i}$ acts as both a compressor and as part of the estimator $\widetilde{\mathbf{W}}_{k}^{i}$ used in (6). After all of the leaf nodes have transmitted their signals, the receiving nodes combine the signals they have received from the leaf nodes with their own sensor signals by

$$
\mathbf{z}_{k q}^{i}=\mathbf{W}_{k k}^{i H} \mathbf{y}_{k}^{i}+\sum_{u \in \mathcal{N}_{k} \backslash q} \mathbf{G}_{k u}^{i H} \mathbf{z}_{u k}^{i}
$$

and forward the result to the single node, $q$, from which they have not received any signal yet, i.e., the neighbor that is one hop closer to the root node. Where $\mathbf{G}_{k u}$ are the components in $\mathbf{G}_{k_{-k}}$, corresponding to the $\mathbf{z}_{u k}$ signal from neighbor node $u$. The fusion process continues until the root node has been reached.

\subsection{Diffusion flow}

The diffusion flow is initiated once the root node receives all the signals from its neighboring nodes. The root node then locally broadcasts to its neighbors the combination of its own sensor signals as well as the signals from all of its neighbors, i.e.,

$$
\mathbf{z}_{k}^{i}=\mathbf{W}_{k k}^{i H} \mathbf{y}_{k}^{i}+\sum_{q \in \mathcal{N}_{k}} \mathbf{G}_{k q}^{i H} \mathbf{z}_{q k}^{i}
$$

This process is repeated up the tree and terminates at the leaf nodes.

\subsection{Receiver feedback cancellation}

In the diffusion flow, when a node transmits a fused signal of its own sensor signals and all z-signals of its neighboring nodes, a node $k$ will receive the signal $\mathbf{z}_{q}$ which contains a component corresponding to node $k$ 's own transmitted signal $\mathbf{z}_{k q}$. In [7] this type of data flow was shown to create a feedback loop which prevents the nodes from converging to their optimal LMMSE estimators.

This feedback signal, however, can be removed by using a socalled receiver feedback cancellation (RFC). In RFC nodes have access to the portion of $\mathbf{G}_{q_{-q}}$ that is applied to their signal during the fusion flow, $\mathbf{G}_{q k}$, which allows the nodes to effectively cancel out their own contribution from the received signals. Node $k$ receives the $\mathbf{G}_{q k}$ that was applied to its signal during the fusion flow from its neighbor and applies this to its received diffusion flow signal (11) as

$$
\mathbf{z}_{q k}^{i}=\mathbf{z}_{q}^{i}-\mathbf{G}_{q k}^{i H} \mathbf{z}_{k q}^{i} .
$$

We note that while this requires an additional data flow of parameters, $\mathbf{G}_{q k}$, these are updated at a much slower rate than the rate of the fusion and diffusion, which must be executed at every instance of new data.

\subsection{Parametrized solution space}

We now define a path, $P_{p_{1} \rightarrow p_{2}}$, which consists of an ordered set of nodes. In Figure 1(a), going from node 1 to node 6 , the path is $P_{1 \rightarrow 6}=\{1,8,2,7,4,6\}$. We also define $\mathbf{G}_{p_{1} \rightarrow p_{2}}$ which follows the previously defined path $P_{p_{1} \rightarrow p_{2}}$, and using the previous example, corresponds to $\mathbf{G}_{1 \rightarrow 6}=\mathbf{G}_{18} \mathbf{G}_{82} \mathbf{G}_{27} \mathbf{G}_{74} \mathbf{G}_{46}$.

Using this definition of $\mathbf{G}_{p_{1} \rightarrow p_{2}}$, the T-DANSE algorithm can therefore be thought of as a parameterization of (4), that at node $k$, effectively corresponds to

$$
\mathbf{W}_{k}=\left[\begin{array}{c}
\mathbf{W}_{11} \mathbf{G}_{k \rightarrow 1} \\
\vdots \\
\mathbf{W}_{K K} \mathbf{G}_{k \rightarrow K}
\end{array}\right] .
$$

Using this parameterization, it was shown in [7] that the optimal es-

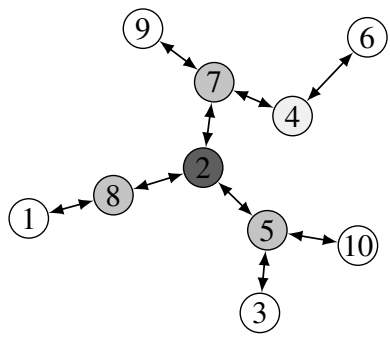

(a) Data flow in original T-DANSE algorithm

Fig. 1. Signal flow in the original and modified T-DANSE algorithm. The red arrows in (b) indicate the fusion flow which is equivalent to the original T-DANSE formulation (a). The blue arrows in (b) indicate the broadcast signal flow from the root node to all other nodes in the WSN.

timators given in (4) are in the solution space defined by (13). Indeed by setting all $\mathbf{G}_{k q}=\overline{\mathbf{A}}_{k q}$ then we automatically have $\mathbf{G}_{k \rightarrow q}=\overline{\mathbf{A}}_{k q}$ for any $k$ and $q$ since $\overline{\mathbf{A}}_{k q}=\overline{\mathbf{A}}_{n q} \overline{\mathbf{A}}_{k n}$ for all $k, q$, and $n$. From (5), we then see that $\hat{\mathbf{W}}_{k}, k=1, \ldots, K$ can be parameterized by (13).

\section{MODIFIED SIGNAL FLOW IN THE T-DANSE ALGORITHM}

In Section 3 the T-DANSE algorithm was described where each node transmits signals to its neighboring nodes in the fusion and diffusion signal flow. However, we see that each non-leaf and non-root node must transmit two signals, one for the diffusion and one for the fusion flow, together with the portion of their node-specific estimators that are applied during the fusion flow, $\mathbf{G}_{k q}$.

\subsection{Root node broadcast signal and feedback cancellation}

In order to reduce the transmission burden at each node we now look to eliminate the diffusion signal flow and allow the root node to broadcast a single signal (11) to all other nodes assuming the root node has a large (virtually infinite) energy budget. An example of the envisaged signal flow is given in Figure 1(b). We note that this method still requires that the $\mathbf{G}_{k q}$ 's be transmitted to neighboring nodes, however this is done over longer time intervals.

We see that if each node receives this signal from the root node it still needs a way to cancel out its own signals contribution in order to arrive at the optimal LMMSE estimators. This can be accomplished in the same manner as the original T-DANSE algorithm, where nodes transmit their $\mathbf{G}_{k q}$ 's to neighboring nodes but now, instead of only being used by a neighboring node, they are multiplied with one another as they are transmitted further up the tree.

We now assume that node $r$ is the root node of the tree and define a path from the root node to a node $k$ as $P_{r \rightarrow k}$. In the proposed diffusion flow each node receives the broadcast signal from the root node and $\mathbf{G}_{\mathrm{r} \rightarrow k}$ that originates at the root node and is a product of all of the $\mathbf{G}_{m n}$ 's (with $n \in N_{m}$ and $m, n \in P_{r \rightarrow k}$ ) between the node and the root node. This differs from the parameter flow given in Section 3.3 where the $\mathbf{G}_{k q}$ 's are only used from neighboring nodes.

Node $k$ then receives the broadcast signal from the root node as well as $\mathbf{G}_{\mathrm{r} \rightarrow k}$, which is applied to the root node broadcast signal as

$$
\mathbf{z}_{r \rightarrow k}^{i}=\mathbf{z}_{r}^{i}-\mathbf{G}_{r \rightarrow k}^{i H} \mathbf{z}_{k q}^{i}
$$

where $\mathbf{z}_{k q}^{i}$ is the signal transmitted by node $k$ in the fusion flow to node $q$ which is one hop closer to the root node. This effectively allows a node to cancel out its own contribution from the broadcast 
signal from the root node. The diffusion flow signal $\mathbf{z}_{k_{-k}}^{i}$, used in the LMMSE estimation problem (6), now consists of $\mathbf{z}_{r \rightarrow k}^{i}$, rather than the signal defined in (11) with corresponding $\mathbf{G}_{k \rightarrow r}$ applied to the root node broadcast signal. Note, that while (12) and (14) are similar, they are only equivalent for the neighbors of the root node.

We see, that despite the modified signal flow, the parametrization still lies in the solution space given in (13), i.e., $\mathbf{G}_{1 \rightarrow 6}=$ $\mathbf{G}_{1 r} \mathbf{G}_{r 7} \mathbf{G}_{74} \mathbf{G}_{46}$ and when setting $\mathbf{G}_{k q}=\overline{\mathbf{A}}_{k q}$,

$\mathbf{G}_{1 \rightarrow 6}=\overline{\mathbf{A}}_{1 r} \overline{\mathbf{A}}_{r 7} \overline{\mathbf{A}}_{74} \overline{\mathbf{A}}_{46}=\overline{\mathbf{A}}_{16}$. Simulations indicate that convergence is reached and that the $\mathbf{G}_{k q}$ 's indeed converge to the $\overline{\mathbf{A}}_{k q}$ 's using the modified signal flow.

\subsection{Reduction in input-output delay and transmission energy}

We note by allowing the root node to broadcast a signal to all other nodes in the WSN, the input-output delay is reduced. This is due to the fact that in the original T-DANSE scheme data must travel between every node in the network, whereas in the modified scheme the longest path data must travel is from the furthest leaf node to the root node plus the single hop broadcast from the root node to all other nodes in the network.

In using the modified scheme, half of the burden of transmission is shifted to the root node. We use a simplified energy model for transmission of signals that is based on the distance between nodes given as

$$
E_{k q}=b \times d_{k q}^{\alpha}
$$

where $d_{k q}$ is the distance between nodes $k$ and $q$, and $\alpha>2$ is a constant $[8,12]$ and $b$ is the number of bits. We denote $E_{k q, F}$ as the energy required for a node $k$ to reach its single neighbor to which a fusion flow signal is transmitted and $b_{s}$ represents the number of bits for signal transmission. We denote $E_{k q, D}$ as the energy required to transmit the diffusion flow signal to all the neighboring nodes of node $k$ (assuming the original T-DANSE algorithm is applied). Since a node transmits the same signal to all of its neighbors in the diffusion flow, the transmission energy required to broadcast is proportional to the largest distance between the node and its neighbors that receive the diffusion flow signal. We also denote $E_{k q, G}$ as the energy required to transmit a nodes $\mathbf{G}_{k q}$ 's to its neighbors in the diffusion flow, which unlike the diffusion signal flow, must be transmitted to each individual neighbor that receives the diffusion flow signal and $b_{c}$ represents the number of bits for the transmission. We denote $E_{k \text {,total }}$ as the total energy used for transmission in the original T-DANSE algorithm, which is given as a summation of all of a nodes energy terms, i.e.,

$$
E_{k, \text { total }}=E_{k l, F}+\sum_{q \in \mathcal{N}_{k} \backslash l}\left(E_{k q, G}+E_{k q, D}\right)
$$

In the modified T-DANSE algorithm we see that for all non-root nodes the right most term in (16), $E_{k q, D}$, vanishes as there is no signal flow in the diffusion flow.

\section{SIMULATIONS}

Monte-Carlo simulations are performed on 100 sample environments where each sample environment has dimensions of $5 \mathrm{~m} \times 5 \mathrm{~m}$. In each simulation, there are $Q=3$ desired source signals randomly distributed throughout the environment where each desired source signals consists of 10000 samples generated from a uniformly distributed random process on the interval [-0.5 0.5]. Each simulated WSN consists of 20 nodes deployed throughout environment. A minimum spanning tree is found during each simulation using Prim's algorithm [13]. Each node has $M_{k}=5$ sensors $^{3}$ that

\footnotetext{
${ }^{3}$ We reiterate that in order to guarantee convergence of the T-DANSE algorithm $M_{k} \geq Q$.
}

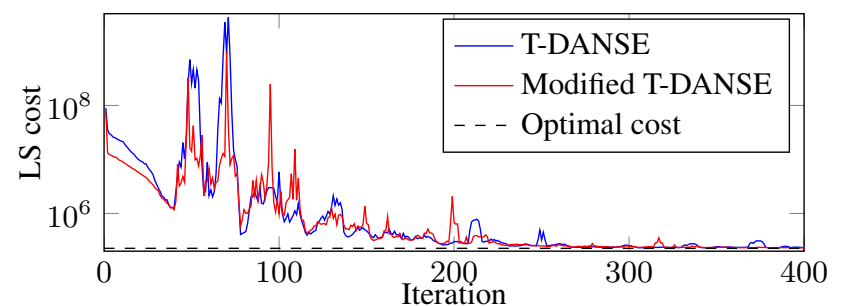

Fig. 2. Average LMMSE of the sum of all nodes for the, TDANSE, modified T-DANSE and optimal LMMSE solution.

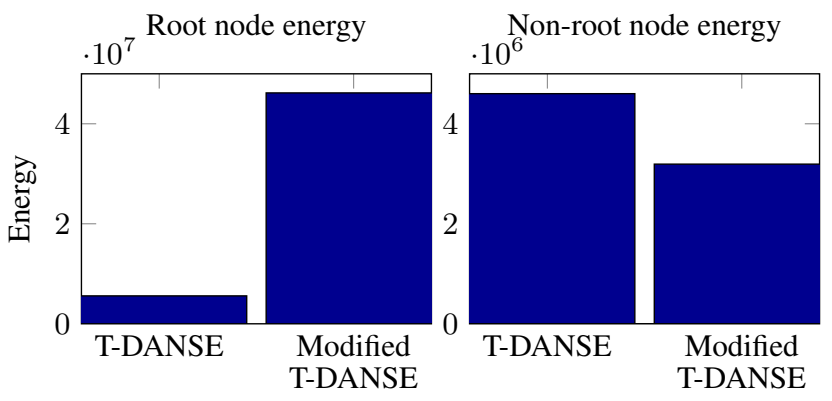

Fig. 3. Average transmission energy of the root node compared to the non-root nodes for the 100 sample environments.

are spaced uniformly around a $10 \mathrm{~cm}$ radius from the center of the node. The coefficients of $\mathbf{A}_{k}$ are proportional to an attenuation factor of $\frac{1}{d}$ where $d$ is the distance between the desired source and the sensor. There are also 5 noise sources which are generated with a similar process as the desired source signals. Spatially uncorrelated white noise, representative of sensor noise, that is half the power of the summed source signals, is added to each sensor. An $\alpha=2.3$ is used to find the energy of each node during the fusion and diffusion flow of signals and a $b_{s}=640$ kbits is used for each transmitted signal from a node and a $b_{c}=576$ bits is used for the transmission of the $\mathbf{G}$ coefficients.

Figure 2 shows the convergence of the average LMMSE of the sum of all of the nodes versus the number of iterations for both the original and modified T-DANSE algorithm compared to the average optimal LMMSE solution. We see that the modified algorithm has similar convergence properties of the original T-DANSE algorithm.

Figure 3 shows the average energy usage for transmission using (16) and the truncated version for non-root nodes for both the original and modified T-DANSE algorithm at the root and non-root nodes. We see that the average energy is reduced for the non-root nodes using the modified broadcast strategy, and that the transmission energy is shifted more to the root node which is in-line with the original goal of the paper as we assume the root node has a virtually infinite energy budget. It should also be noted that the average transmission energy is reduced in each node as given in Figure 3, therefore the total reduction in energy over the WSN for all non-root nodes is 19 times the reduction in transmission energy.

\section{CONCLUSIONS}

A modified T-DANSE algorithm was proposed which was able to reduce the overall input-output delay of the system and shift the energy usage to that of the root node. This modified algorithm lends itself readily to tree topologies in which a base station has larger broadcast abilities as well as larger energy resources. The proposed signal flow was demonstrated to be equivalent in terms of signal estimation to that of the original T-DANSE algorithm in that every node in the WSN was still able to converge to the centralized solution. 


\section{REFERENCES}

[1] Y. Li, M.T. Thai, and W. Wu, Wireless Sensor Networks and Applications, Signals and communication technology. Springer, 2008.

[2] S. Haykin and K.J.R. Liu, Handbook on Array Processing and Sensor Networks, IEEE, Hoboken, N.J. Wiley, Piscataway, NJ, 2009.

[3] J. Yick, M. Biswanath, and D. Ghosal, "Wireless sensor network survey," Computer Networks, vol. 52, no. 12, pp. 2292 2330, 2008.

[4] G. Călinescu and S. Tongngam, "Relay nodes in wireless sensor networks," in 3rd Int. Conf. on Wireless Algorithms, Systems, and Applications (WASA), 2008, pp. 286-297.

[5] X. Cheng, D.Z. Du, L. Wang, and B. Xu, "Relay sensor placement in wireless sensor networks," Wireless Networks, vol. 14, no. 3, pp. 347-355, June 2008.

[6] A. Bertrand and M. Moonen, "Distributed adaptive nodespecific signal estimation in fully connected sensor networks - part I: Sequential node updating," IEEE Trans. Signal Process., vol. 58, no. 10, pp. 5277 -5291, oct. 2010.

[7] A. Bertrand and M. Moonen, "Distributed adaptive estimation of node-specific signals in wireless sensor networks with a tree topology," IEEE Trans. on Signal Process., vol. 59, no. 5, pp. 2196-2210, May 2011.

[8] A.K. Das, R.J. Marks II, M. El-Sharkawi, P. Arabshahi, and A. Gray, "Minimum power broadcast trees for wireless networks: integer programming formulations," in 22nd Annu. Joint Conf. of the IEEE Computer and Commun. (INFOCOM), 2003, vol. 2, pp. 1001-1010.

[9] I. Kang and R. Poovendran, "Broadcast with heterogeneous node capability [wireless ad hoc or sensor networks]," in Global Telecommunications Conf. (GLOBECOM), 2004, vol. 6, pp. 4114-4119.

[10] Y-H. Song, C. Suh, and T-G. Kwon, "Design and implementation of efficient base station for wireless sensor networks," in 5th IEEE Conf. on Sensors (ICSENS), 2006, pp. 726-729.

[11] T. Lee, Y. Yang, K. Shin, and M. Park, "An energy-efficient uni-scheduling based on s-mac in wireless sensor network," in 1st Int. Conf. on High Performance Computing and Commun. (HPCC), 2005, pp. 293-304.

[12] X. Chen and N.C. Rowe, "Saving energy by adjusting transmission power in wireless sensor networks," in Global Telecommunications Conf. (GLOBECOM), 2011, pp. 1-5.

[13] T. H. Cormen, C. Stein, R. L. Rivest, and C. E. Leiserson, Introduction to Algorithms, McGraw-Hill Higher Education, 2nd edition, 2001. 